\title{
Coordinating Efforts to Define Marine Plankton Imagery Data and Metadata Best Practices and Standards
}

\author{
Patricia Martin-Cabrera $\ddagger$, Fabien Lombard§, Jean-Olivier Irisson§, Lars Stemmann§, Klas O. Möllerl, \\ Markus Lindh", Veronique Creach\#, Lennert Schepers ${ }^{\ddagger}$ \\ ‡ Flanders Marine Institute, Ostend, Belgium \\ $\S$ Institut de la Mer de Villefranche, CNRS - Sorbonne Université, Villefranche-sur-mer, France \\ | Institute of Coastal Research, Helmholtz-Zentrum Geesthacht, Geesthacht, Germany \\ II Swedish Meteorological and Hydrological Institute, SMHI, Västra Frölunda, Sweden \\ \# Centre for Environment, Fisheries and Aquaculture Science, CEFAS, Lowestoft, United Kingdom
}

Corresponding author: Patricia Martin-Cabrera (patricia.cabrera@vliz.be)

Received: 24 Sep 2020 | Published: 29 Sep 2020

Citation: Martin-Cabrera P, Lombard F, Irisson J-O, Stemmann L, Möller KO, Lindh M, Creach V, Schepers L

(2020) Coordinating Efforts to Define Marine Plankton Imagery Data and Metadata Best Practices and

Standards. Biodiversity Information Science and Standards 4: e58932. https://doi.org/10.3897/biss.4.58932

\begin{abstract}
"Imagery data" can be referred as qualitative and quantitative information from a collection of images. Imaging systems are used more and more frequently in the marine domain to generate huge amounts of imagery data. For example, automatic image classification is used to determine the abundance, size and biomass of plankton communities. In addition, the recent advances of imaging sensors and the growing datasets, highlight the importance of the management and storage capacity of these data. Thus, establishing data standards, optimized data flows and quality control procedures will promote the ability to make these datasets findable, accessible, interoperable and reusable (FAIR principles).
\end{abstract}

At the moment, there are a number of online open-access databases that collect marine biodiversity data, such as the Ocean Biodiversity Information System ( $\underline{\mathrm{OBIS}}$ ), and more specialized in plankton observations such as the COPEPOD for plankton biomass and ECOTAXA for taxonomic annotation of plankton images. However, they currently lack relevant standards to link metadata of the images. 
International biodiversity data standards, such as Darwin Core (DwC), are already widely used in OBIS and the Global Biodiversity Information Facility (GBIF). OBIS, has recently adopted the OBIS-ENV-DATA, a format that follows the DwC-Archive (DwC-A) standard, consisting of a DwC Event table in combination with an Occurrence an extended Measurement or Fact table. This structure enables the linkage of quantitative and qualitative properties to both sampling events and species occurrences. It also includes additional fields for property standardization, such as the BODC (British Oceanographic Data Centre) controlled vocabularies, the World Register of Marine Species (WoRMS), which provides crucial quality control support for taxonomic data, and Marine Regions, a database that provides standardized marine georeferenced place names and areas. However, there are no clear guidelines on how to include imagery metadata and derived data in OBIS-ENV-DATA.

During this TDWG 2020 symposium, we would like to present and discuss our ongoing work to establish best practices and standardized protocols for imaging data and metadata acquired by a large spectrum of bio-optic sensors. Furthermore, we highlight how the integration of the metadata will flow to existing biodiversity data portals, the European EMODnet Biology portal, (EurOBIS) and OBIS.

\section{Keywords}

imagery data, data standardization, Darwin Core Archive, species occurrence, plankton

\section{Presenting author}

Patricia Martin-Cabrera

\section{Presented at}

TDWG 2020

\section{Funding program}

H2020-INFRAIA

\section{Grant title}

Joint European Research Infrastructure of Coastal Observatories: Science, Service, Sustainability- JERICO-S3 (project 871153) 


\section{Hosting institution}

Flanders Marine Institute (VLIZ) 